\title{
Communication
}

\section{Effect of the marine $\beta$-carboline alkaloid Manzamine A on RSK1 vs RSK2 inhibition: a biochemical and computational study}

\section{Alejandro M.S. Mayer ${ }^{1 *}$, Mary L. Hall ${ }^{1}$, Joseph Lach $^{1}$, Jonathan Clifford ${ }^{1}$, Kevin Chandrasena $^{1}$, Caitlin Canton ${ }^{1}$ and Maria Kontoyianni ${ }^{2}$}

1 Department of Pharmacology, College of Graduate Studies, Midwestern University, $55531^{\text {st }}$ Street, Downers Grove, Illinois 60515, USA; E-Mails: mhall1@ midwestern.edu (M.L.H.); joseph.m.lach@gmail.com (J.L.); clifford07@gmail.com (J.C.); kschandras@gmail.com (K.C.); canto030@umn.edu (C.C.)

2 Department of Pharmaceutical Sciences, Southern Illinois University Edwardsville, Edwardsville, Illinois 62026-2000, USA; E-Mail: $\underline{\text { mkontoy@ siue.edu }}$

* Author to whom correspondence should be addressed; E-Mail: amayer@ midwestern.edu Tel.: (630) 515-6951; Fax: (630) 515-6295.

\begin{abstract}
Manzamines are complex polycyclic marine-derived $\beta$-carboline alkaloids with reported anticancer, immunostimulatory, anti-inflammatory, antibacterial, antiviral, antimalarial, neuritogenic, hyperlipidemia and atherosclerosis suppression bioactivities, putatively associated with inhibition of glycogen synthase kinase-3, cyclin-dependent kinase 5, and vacuolar ATPases. We hypothesized that additional and yet undiscovered molecular targets might be associated with Manzamine A (MZA) reported pharmacological properties. We report herein for the first time to our knowledge that MZA inhibited a 90kDa ribosomal protein kinase S6 (RSK1) when screened against a panel of 30 protein kinases. Furthermore in vitro RSK kinase assays demonstrated a 10-fold selectivity in potency of MZA against RSK1 versus RSK2. MZA's differential binding and selectivity toward the two isoforms is also supported by computational docking experiments. Specifically, the RSK1-MZA (N- and Ctermini) complexes appear to have stronger interactions and preferable energetics contrary to the RSK2-MZA ones. In addition, our computational strategy suggests that MZA binds to the N-terminal kinase domain of RSK1 rather than the C-terminal
\end{abstract}


domain. RSK is a vertebrate family of cytosolic serine-threonine kinases that act downstream of the ras-ERK1/2 (extracellular-signal-regulated kinase 1/2) pathway, which phosphorylates substrates shown to regulate several cellular processes including growth, survival and proliferation. Consequently, our findings have lead us to hypothesize that MZA and the 80 currently known manzamine-type alkaloids isolated from several sponge genera, may have novel pharmacological properties.

Keywords: MZA, Manzamine A; CTKD, C-terminal kinase domain; ras-ERK1/2 (extracellular-signal-regulated kinase 1/2) pathway; RSK1, 90kDa ribosomal protein S6 kinase 1; RSK2, 90kDa ribosomal protein S6 kinase 2; NTKD, N-terminal kinase domain

\section{Introduction}

Marine biological and chemical diversity continues to demonstrate great potential to contribute novel pharmacology for multiple therapeutic categories [1]. Although the current marine pharmaceutical clinical pipeline consists mainly of compounds developed for cancer chemotherapy [2], several marine natural products have been shown to target a range of protein kinases as well [3].

First reported in the Okinawan sponge genus Haliclona [4], the manzamine class consists of complex polycyclic marine-derived alkaloids which possess a fused and bridged tetra- or pentacyclic ring system attached to a $\beta$-carboline moiety, and currently includes more than 80 additional manzamine-type alkaloids isolated from several sponge genera [5]. Manzamines exhibit a range of bioactivities: anticancer [6], immunostimulatory [7], anti-inflammatory [8,9], antibacterial [9], antiviral [9,10], antimalarial [11], neuritogenic [12] and hyperlipidemia and atherosclerosis suppression in vivo [13]. Furthermore, several molecular targets have been reported 
for the manzamines, namely glycogen synthase kinase-3 (GSK-3), cyclin-dependent kinase 5 (CDK5) [14], and vacuolar ATPases [15]. We hypothesized that additional and yet undiscovered molecular targets might be associated with Manzamine's pharmacological properties.

The $90 \mathrm{kDa}$ ribosomal S6 Kinase or RSK is a vertebrate family of cytosolic serine-threonine kinases that contains four homologous isoforms namely RSK1-4, which act downstream of the ras-ERK1/2 (extracellular-signal-regulated kinase 1/2) pathway [16]. RSK1 and RSK2 adult and embryonic tissue expression has been investigated, with RSK1 found in lung, kidney, pancreas and brain (cerebellum and microglia) [16-18], while RSK2 is more abundant in skeletal muscle, heart, pancreas and brain (neocortex, hippocampus, and cerebellum)[16]. RSK kinases are composed of two functional kinase catalytic domains: the C-terminal kinase domain (CTKD) that belongs to the calcium and calmodulin-regulated kinases CamK family, is phosphorylated by ERK1/2, and activates the N-terminal kinase domain (NTKD) that belongs to the protein kinase A, G, and C (AGC) family [16]. NTKD has been shown to phosphorylate several substrates, and in turn regulates several cellular processes including growth, survival, and proliferation [16]. Interestingly, loss of RSK2 function causes a rare form of mental retardation known as CoffinLowry syndrome [19], while sustained activation of RSKs appears to be linked to cancer [20,21]. RSK inhibitors used in preclinical studies include two NTKD-interacting inhibitors at the ATPbinding site, namely the reversible dihydropteridinone BI-D1870 [22], and the kaempferol glycoside SL0101 [23], and the pyrrolopyrimidine FMK, a CTKD-interacting irreversible inhibitor that binds to the ATP-binding pocket of RSK [24].

Herein we report that MZA inhibited the 90kDa RSK1 when screened against a panel of 30 protein kinases. In vitro kinase assays demonstrated a 10-fold selectivity in potency between RSK1 and RSK2 (IC50 values of $15.01 \mu \mathrm{M}$ and $108.4 \mu \mathrm{M}$, respectively). Furthermore, MZA is 
predicted to bind to the ATP-binding pocket of NTKD of RSK1 and its selectivity towards RSK1 versus RSK2 are supported by our computational studies reported herein.

\section{Results}

\subsection{Effect of manzamine A on a 30 protein kinases screening array}

MZA (Figure 1) was screened using a panel of 30 protein kinases as described $[25,26]$.

Figure 1: Manzamine A (MZA) is an indole-derived alkaloid isolated from the marine sponge Haliclona sp. [4].

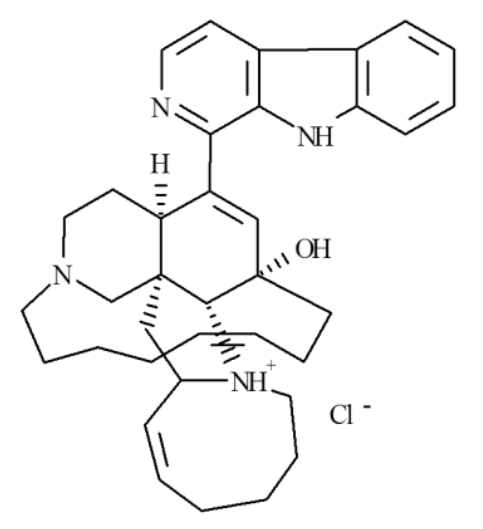

As shown in Table 1, MZA $(1 \mu \mathrm{M})$ reduced the activity of rat p90 ribosomal S6 kinase 1 or RSK1 by $68 \%$. Furthermore, MZA showed no significant inhibitory effect on the other 29 kinases which were part of the University of Dundee protein kinase enzyme panel. 
Table 1: Effect of MZA on a 30 protein kinase enzyme panel

I.

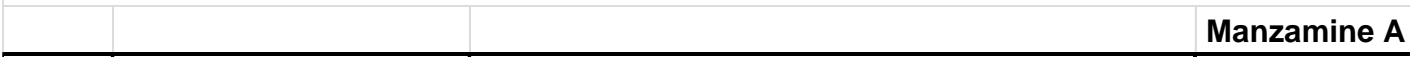

\begin{tabular}{|c|c|c|c|}
\hline & Protein Kinase & Full Name & $1 \mu M^{\star}$ \\
\hline 1 & MKK1 & MAPK kinase & 90 \\
\hline 2 & MAPK2/ERK2 & mitogen-activated protein kinase & 93 \\
\hline 3 & JNK1a1 & c-Jun N-terminal kinase & 80 \\
\hline 4 & $\mathrm{SAPK} 2 \mathrm{a} / \mathrm{p} 38$ & stress-activated protein kinase- $2 \mathrm{a}$ & 75 \\
\hline 5 & $\mathrm{SAPK} 2 \mathrm{~b} / \mathrm{p} 38 \mathrm{~b} 2$ & stress-activated protein kinase- $2 \mathrm{~b}$ & 84 \\
\hline 6 & SAPK3/p38g & stress-activated protein kinase-3 & 100 \\
\hline 7 & SAPK4/p38d & stress-activated protein kinase- 4 & 98 \\
\hline 8 & МАРКАР-K1a (RSK-1) & MAPK-activated protein kinase-1a & 32 \\
\hline 9 & MAPKAP-K2 & MAPK-activated protein kinase-2 & 92 \\
\hline 10 & MSK1 & mitogen and stress-activated protein kinase-1 & 73 \\
\hline 11 & PRAK & p38-regulated/activated kinase & 93 \\
\hline 12 & PKA & cyclic AMP-dependent protein kinase & 90 \\
\hline 13 & $\mathrm{PKCa}$ & protein kinase $\mathrm{C}$ & 90 \\
\hline 14 & PDK1 & 3-phosphoinositide-dependent protein kinase-1 & 105 \\
\hline 15 & $\mathrm{PKBa}$ & protein kinase B & 75 \\
\hline 16 & SGK & serum and glucocorticoid-induced kinase & 61 \\
\hline 17 & S6K1 & p70 ribosomal protein S6 kinase & 82 \\
\hline 18 & GSK3b & glycogen synthase kinase- $3 \mathrm{~b}$ & 73 \\
\hline 19 & ROCK-II & Rho-dependent protein kinase & 105 \\
\hline 20 & AMPK & AMP-activated protein kinase & 97 \\
\hline 21 & CHK1 & checkpoint kinase-1 & 86 \\
\hline 22 & $\mathrm{CK} 2$ & casein kinase-2 & 83 \\
\hline 23 & PHK & phosphorylase kinase & 98 \\
\hline 24 & LCK & lymphocyte kinase & 71 \\
\hline 25 & CSK & C-terminal Src kinase & 89 \\
\hline 26 & CDK2/cyclin A & cyclin-dependent kinase 2-cyclin A complex & 107 \\
\hline 27 & DYRK1A & Dual tyrosine phosphorylated and regulated kinase $1 \mathrm{~A}$ & 82 \\
\hline 28 & CK1 & Casein kinase 1 & 91 \\
\hline 29 & NEK 6 & NIMA-related protein kinase 6 & 74 \\
\hline 30 & NEK2a & NIMA-related protein kinase $2 \mathrm{a}$ & 96 \\
\hline
\end{tabular}

* Mean \% Activity Remaining

\subsection{Effect of manzamine A on RSK1 and RSK2}

As shown in Figure 2, MZA showed selective moderate inhibition of RSK1 [ $\left.\mathrm{IC}_{50}=15.01 \mu \mathrm{M}\right]$ vs. RSK2 $\left[\mathrm{IC}_{50}=108.4 \mu \mathrm{M}\right]$ in 3 independent experiments. In contrast, and as previously reported, BID1870 potently inhibited RSK1 and $\mathrm{RSK} 2\left[\mathrm{IC}_{50}=0.016\right.$ and $0.008 \mu \mathrm{M}$, respectively] but showed no selectivity towards either RSK1 or RSK2 [22]. 
Figure 2: Effect of MZA and BI-D1870 on (A) RSK1 and (B) RSK2 activity.

RSK1 and RSK2 kinase assays were conducted as described in Materials and Methods.

\section{A. RSK1}

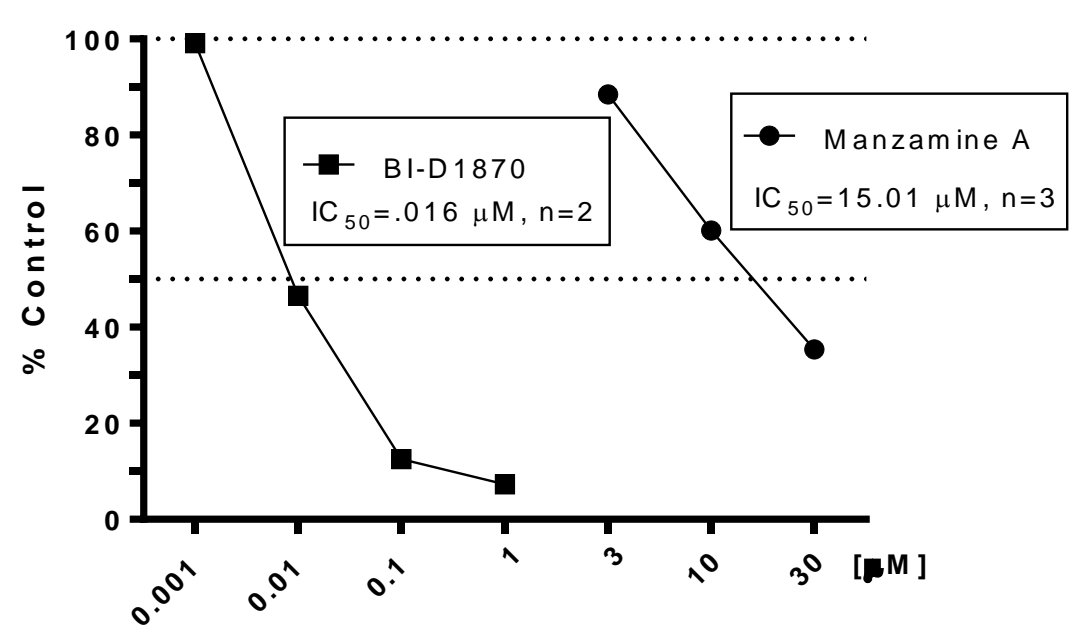

\section{B. RSK2}

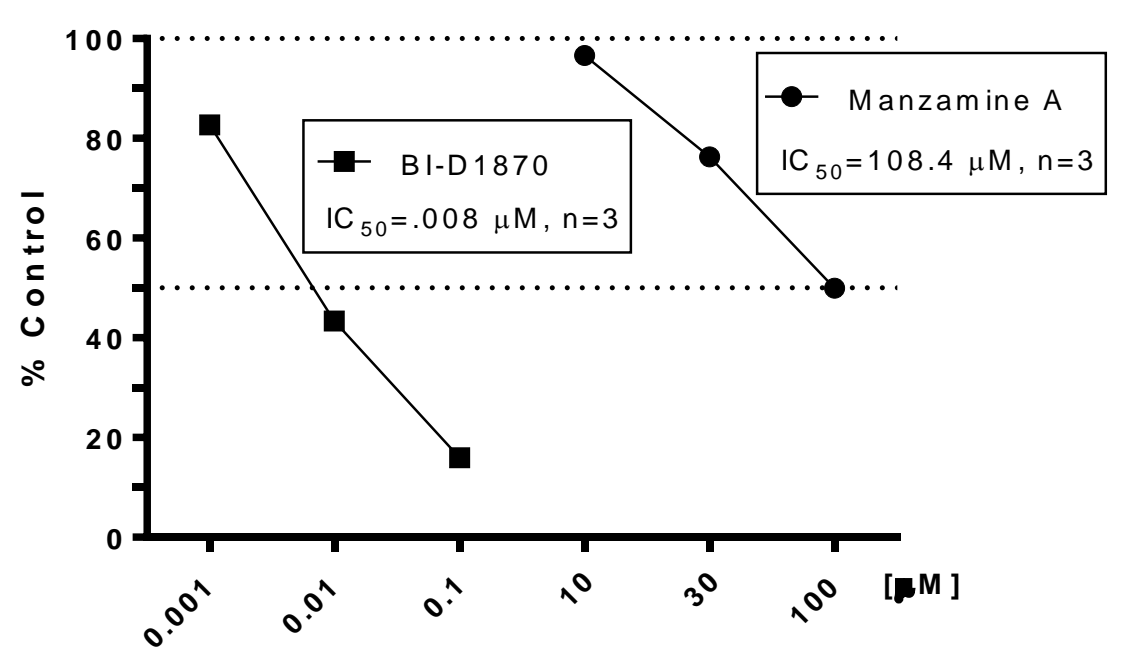




\subsection{Computational Studies}

Superimposition of respective NTKDs and CTKDs of crystal RSK1 and RSK2 structures (see Methods) to identify 'a' representative structure for subsequent docking experiments was undertaken. Selected structures are presented in Table 2.

\begin{tabular}{|cc|cc|}
\hline \multicolumn{2}{|c|}{ Table 2. Crystal Structures Employed in the Study } \\
\hline \multicolumn{2}{|c|}{ RSK1 } & \multicolumn{2}{|c|}{ RSK2 } \\
\hline$C T D^{a}$ & $N T D^{b}$ & $C T D$ & $N T D$ \\
\hline 3RNY & 2Z7Q & 4D9T & 3G51 \\
\hline
\end{tabular}

${ }^{\mathrm{a}} \mathrm{C}$-terminal domain, ${ }^{\mathrm{b}} \mathrm{N}$-terminal domain.

The active sites of NTKD and CTKD RSK1 (PDB entries 2Z7Q and 3RNY, respectively) with amino acids reportedly involved in binding interactions are depicted in Figure 3. ${ }^{1,5}$

Figure 3: (A) ATP-binding site in the N-terminal domain of RSK1 with the AMP-PCP ligand shown in red. (B) C-terminal domain of RSK1 with ATP-binding site residues depicted by atomtype, while green residues are reflective of amino acids that constrain Glu 496 in a favorable conformation pointing towards the ATP-binding cleft.

A

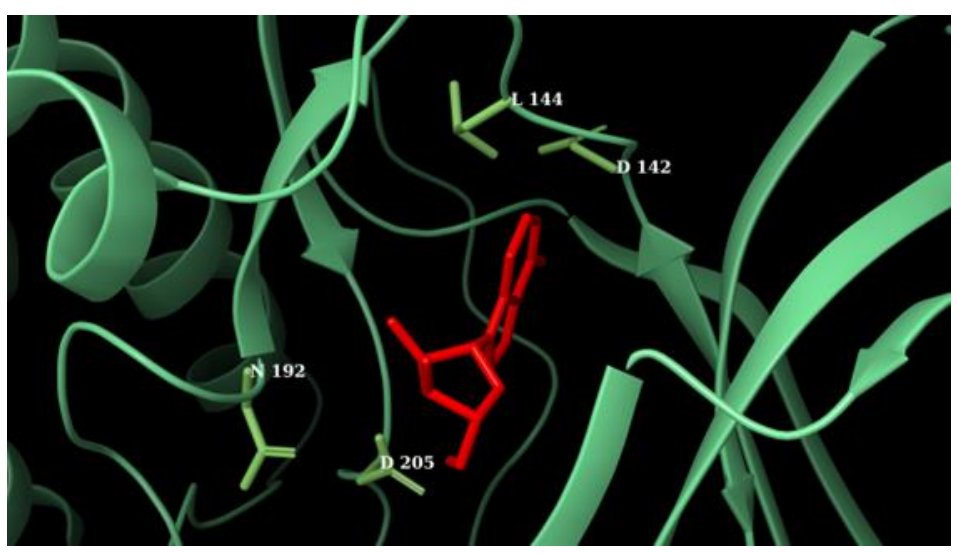




\section{B}

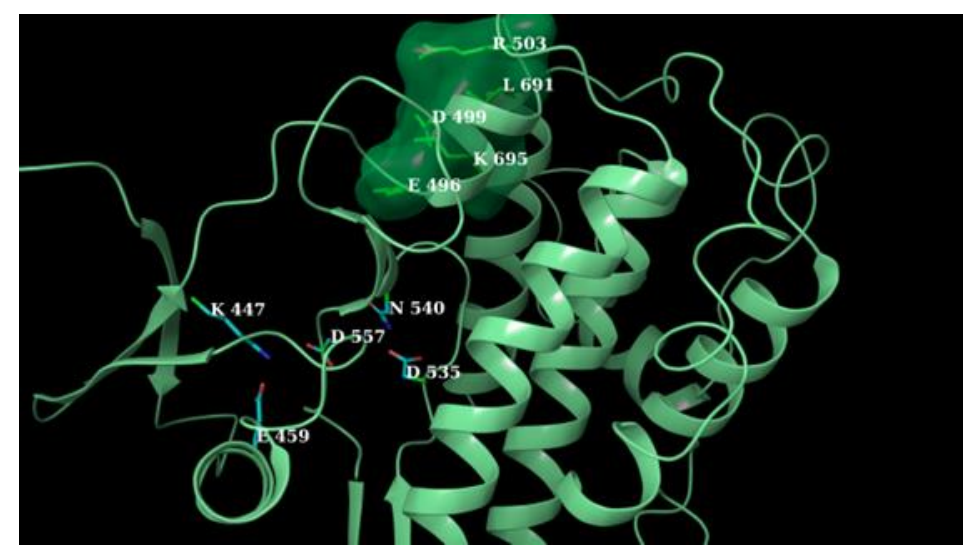

Comparison of the binding pockets in either terminus of RSK1 and RSK2 was performed by overlaying the selected CTKD of RSK1 (PDB 3RNY) onto the CTKD of RSK2 (PDB 4D9T). Figure 4 depicts the equivalent amino acids in the ATP-binding clefts of both structures. Similarly, the overlay of the NTKDs of RSK1 and RSK2 is presented in Figure 5.

Figure 4: Corresponding amino acids in the ATP-binding pockets of the C-termini of RSK1 (green) and RSK2 (orange). Residues in RSK1 are labelled yellow, while white labels designate RSK2 residues.

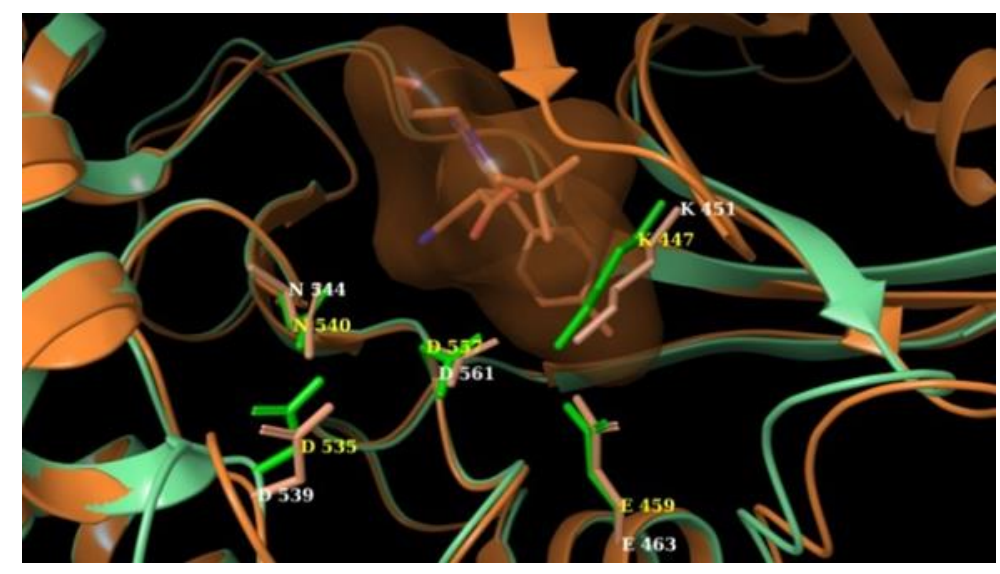


Figure 5: The NTKDs of RSK1 (green, white labels) and RSK2 (orange, yellow labels). Only the ribbons of RSK1 are displayed. Bound ligand in the NTKD RSK1 is depicted in red, while the surface of the binding cleft of RSK2 is shown with its respective bound ligand in cyan.

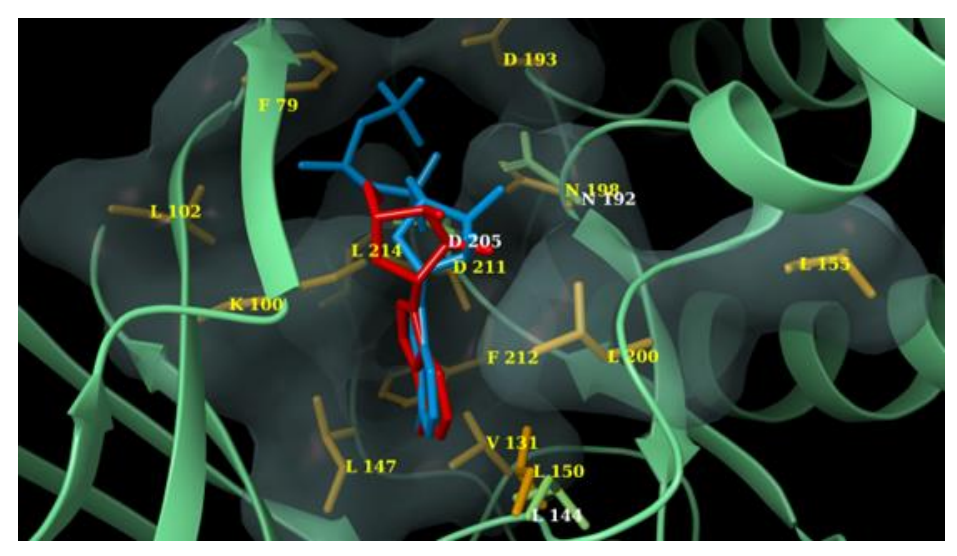

Because we had no prior knowledge as to whether MZA preferentially binds to the one terminus over the other in RSK1, we superimposed the two termini of RSK1. Figure 6A shows the overall NTKD and CTKD RSK1 crystal structures overlayed with the amino acids in the ATP binding pocket of CTKD RSK1 labelled. It should be pointed out there is notable similarity. Figure 6B shows a comparison of the active sites.

Figure 6: (A) Overall topologies of the NTKD (green ribbons) and CTKD (orange, white labels) of RSK1 superimposed. The surface of the ligand in CTKD is in purple. (B) Close-up view of the binding clefts. Amino acids in NTKD are labelled in yellow. All but one residue (Glu 459) thought to be catalytic in CTKD have equivalent ones in NTKD. Surfaces of residues are also depicted in orange (CTKD) and green (NTKD). 


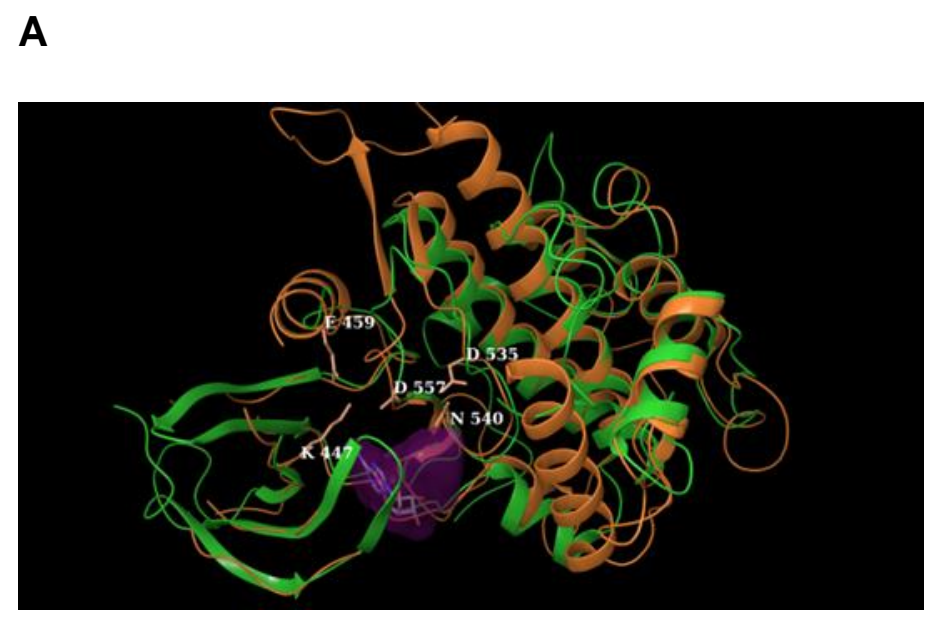

B

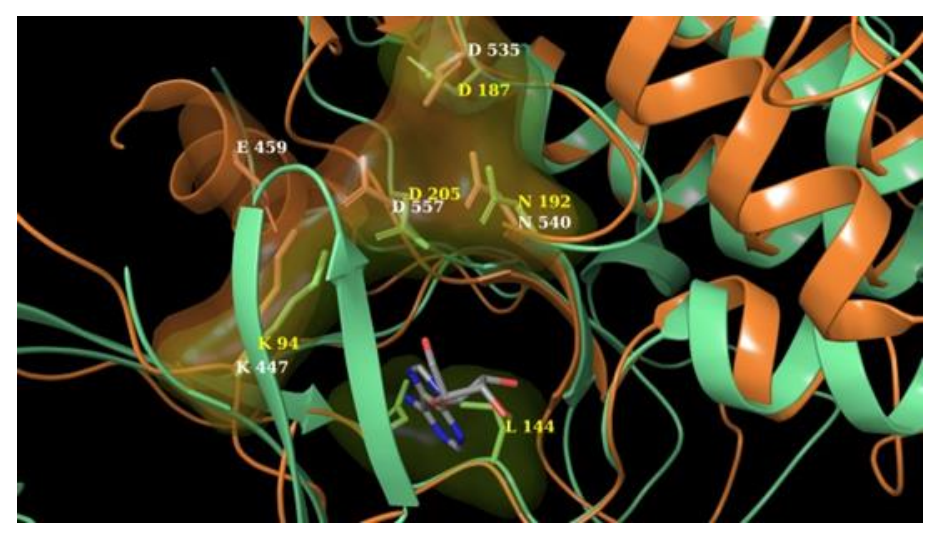

\section{Discussion}

The RSK family of proteins consists of four isoforms that are highly homologous, with the exception of the $\mathrm{N}$ - and C-terminal sequences where divergence is observed. RSKs can receive signals to their CTKD and in turn will transmit an activating signal to their NTKD. The two catalytic domains are connected via a conserved linker of approximately 100 amino acids. As noted earlier, NTKD belongs to the AGC family and is responsible for substrate phosphorylation. CTKD is homologous with the $\mathrm{Ca}^{2+} /$ calmodulin-dependent kinase family and responsible for NTKD's activation via authophosphorylation. 
Although the search for novel RSK inhibitors is ongoing, to our knowledge there is no RSK isozyme specific inhibitor. The RSK reversible dihydropteridinone BI-D1870 [22], and kaempferol glycoside SL0101 [23] inhibit the NTKD ATP-binding site with comparable potency. Similarly, the pyrrolopyrimidine FMK is a CTKD-irreversible inhibitor that binds to the ATPbinding pocket of RSK [24].

Our current study suggests that MZA is a moderate yet selective inhibitor of the RSK1 isozyme based on the biochemical evidence. Thus, we set out to explore computationally MZA's potential binding in order to shed light on the observed selectivity towards RSK1 versus RSK2. Because multiple crystal structures of CTKD and NTKD of both RSK1 and RSK2 have been reported in the literature, we were not certain whether MZA binds to the $\mathrm{N}$ - or C-terminal domains. Consequently, we opted to perform docking experiments toward both NTKD and CTKD of the two isoforms in order to: 1) Rationalize the observed selectivity between the two isozymes and, 2) Identify the plausible preferential binding for one domain over the other.

At first, we superimposed the NTKDs of all crystal RSK1, CTKDs of RSK1, and repeated with an overlay of the NTKDs and CTKDs of RSK2 in order to identify representative structures. Table 2 shows the structures selected for all subsequent work. It should be noted that we favored the active conformation of NTKD RSK2 (PDB 3G51) [27] because we felt it would be more informative regarding the conformational transitions of the domain. Furthermore, even though the CTKD of RSK1 is an apo structure, its ATP-binding cleft has been described [28].

Figure 4 depicts the equivalent amino acids in the ATP-binding clefts of both RSK1 and RSK2 structures (CTKD of RSK1 PDB 3RNY and CTKD of RSK2 PDB 4D9T). It should be pointed out that Glu 496 is constrained in a specific orientation within the ATP-binding cleft of 3RNY, with Glu 500 of 4D9T being the corresponding residue (see Figure 4 and Tables 3b) [28]. A similar 
overlay was undertaken for the NTKDs of RSK1 and RSK2 (Figure 5 and Table 3a). It can be seen that the ATP-binding cleft of RSK1 consists of Asp 142, Asp 205, Leu 144, and Asn 192, as reported by Ikuta et al.[29]. The active conformation of RSK2 is lined by polar charged residues (Asn 198, Asp 193, Lys 100, and Asp 211), along with a hydrophobic patch including Lys 100, Phe 79, Leu 101, Lys 216, Leu 214 [27] Identified amino acids lining up respective binding pockets were employed in all subsequent docking experiments.

Because MZA is shown herein to be selective towards RSK1 over RSK2, and since we have no prior knowledge as to whether it preferentially binds to the one terminus over the other, we superimposed the two termini of RSK1. Figure 6A shows the overall NTKD and CTKD RSK1 crystal structures overlayed with the amino acids in the ATP binding pocket of CTKD RSK1 labelled. In comparing the two topologies, there is a notable similarity, in that with the exception of the N-lobe consisting of a five-stranded antiparallel $\beta$ sheet and a missing helix corresponding to $\alpha \mathrm{E}$ of $\mathrm{CTKD}$, the remaining structures superimpose rather well. Figure $6 \mathrm{~B}$ shows a comparison of the active sites. All but one (Glu 454) of the CTKD amino acids correspond to similar type residues in NTKD. Specifically, Lys 447, Asp 557, Asp 535, and Asn 540 of CTKD are equivalent with Lys 94, Asp 205, Asp 187, Asn 192 of NTKD. This appears to be in agreement with Fisher and Blenis who provided evidence that both domains are active and that NTKD is responsible for phosphotransferase activity, whereas CTKD is catalytically active [30].

Docking of MZA into the CTKD of RSK1 revealed strong hydrogen bonding and pi-stacking interactions (Figure 7). Specifically, the hydroxyl hydrogen bonds with Asp 557 and Arg 454, while the nitrogen of aza-cyclooctene forms a hydrogen bond with Lys 537. Finally, aliphaticaromatic interactions are observed between Phe 560 and the polycyclic moiety of MZA. 
Figure 7: Docked pose of Manzamine A into the CTKD of RSK1. Amino acids forming binding interactions with the ligand are shown in blue and labelled. Catalytic residues reported in the literature are shown in dark purple sticks for reference in regards to Manzamine's positioning within the cleft.

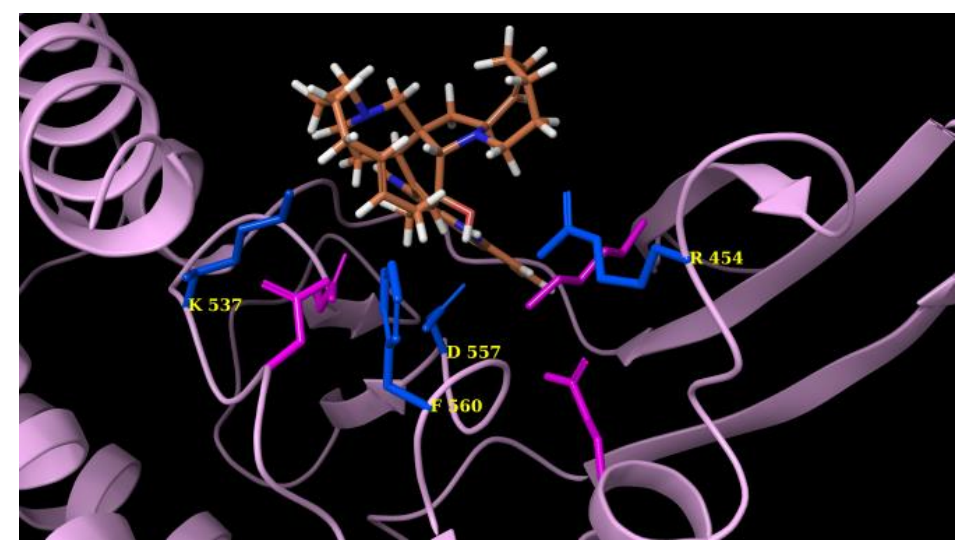

It should be mentioned that in contrast, the predicted binding mode of Manzamine with CTKD of RSK2 (Figure 8) suggests fewer and weaker interactions with the binding pocket, which in turn could account for the observed higher affinity of the compound for RSK1. The hydroxyl group is oriented toward the backbone peptide bond nitrogen of Val 300, while the nitrogen interacts with the carboxylate of Glu 500. The remaining interactions are mostly hydrophobic in nature and no aryl-alkyl interactions are predicted, since Phe 564 (equivalent to 560) is far from the pose.

Figure 8: Predicted pose of Manzamine A into the CTKD of RSK2. Amino acids forming bonding interactions are depicted in stick and labelled. 


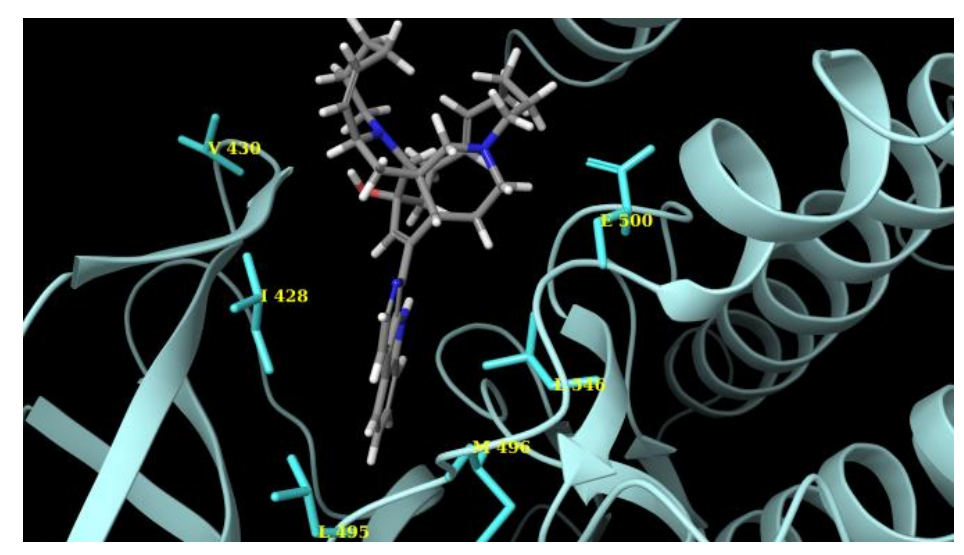

Similarly, we docked MZA into the NTKDs of both RSK1 and RSK2, as described in the preceding sections. We observed an orientation much deeper into the cleft in RSK1 and more interactions than in RSK2. Specifically, the hydroxyl group hydrogen-bonds with the backbone nitrogen of Gln 70, while the $\mathrm{N}$ of the aza-cyclooctene is in close proximity with Asp 148. Leucines 194 and 68 form a hydrophobic patch around the aza-carbazole, while aryl-alkyl interactions are observed with Phe 150 (Figure 9).

Figure 9: Docked orientation of Manzamine A into the NTKD of RSK1. Amino acids involved in bonding interactions are in blue with yellow labels. Additional residues reported in the respective crystal structure are shown in stick (pink).

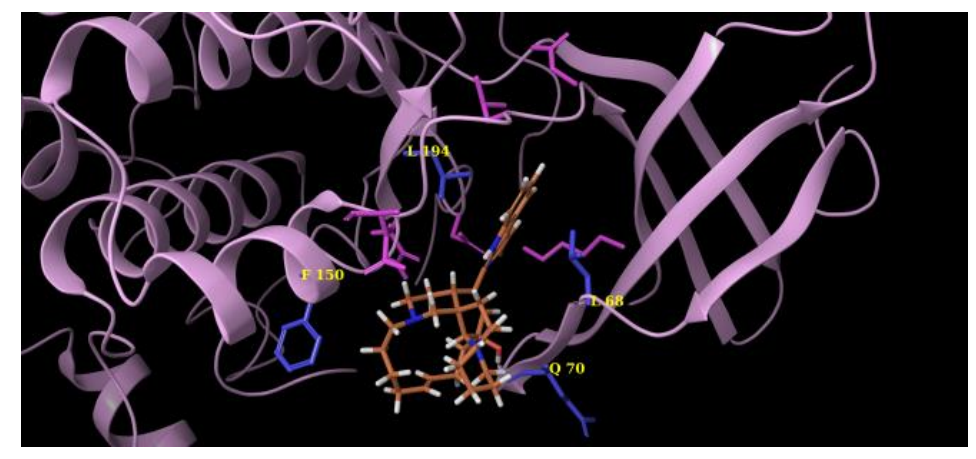


When MZA was docked into the NTKD of RSK2, limited interactions were observed. The hydroxyl interacts with Asp 154, and the secondary amine forms a hydrogen bond with the backbone of Leu 74. $\pi-\pi$ Stacking interactions are observed between carbazole and Phe 149 (see Figure 10).

Figure 10: Predicted orientation of Manzamine A into NTKD of RSK2. Amino acids interacting with the ligand are labelled.

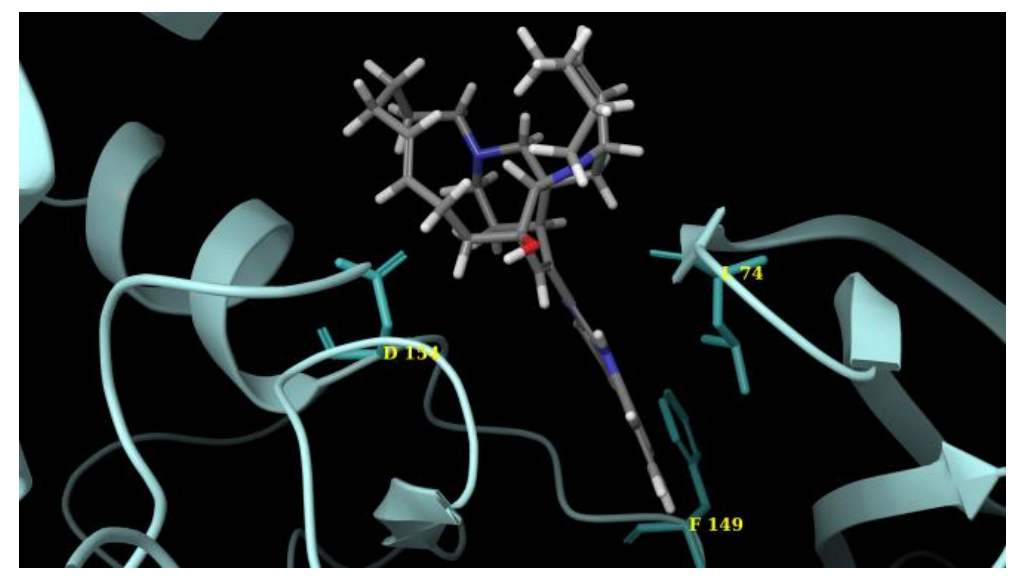

Consequently, it seems that MZA forms a tighter complex with RSK1 and is positioned deeper in the pocket contrary to RSK2. Consequently, using docking experiments coupled with visual comparisons of reported crystal structures we have been able to explain observed binding affinities and selectivity of MZA towards RSK1. Another point of concern was whether we could predict the ligand's binding to NTKD or CTKD. Because the MZA-NTKD RSK1 complex appears to be more stable due to stronger interactions with the surrounding amino acid residues in the ATPbinding pocket, in contrast to the MZA-CTKD RSK1 complex, we suggest that MZA preferentially binds to NTKD. Furthermore, an examination of the predicted binding energies of the NTKD and CTKD complexes with MZA (emodels of -62.132 and -55.497 , respectively) support the notion that MZA shows a preference towards the NTKD of RSK1. 
In conclusion, we report herein an integrated biochemical and computational study investigating the marine-derived alkaloid MZA. We show that MZA selectively inhibits RSK1 versus RSK2 and predict that it binds to its N-terminal kinase domain. To our knowledge, this is the first report of RSK1 as a new macromolecular target of MZA, a finding that extends the pharmacology of the manzamine-type alkaloids.

\section{Materials and Methods}

\subsection{Materials}

Manzamine A (MZA) (Figure 1) was isolated from a marine sponge species of the genus Haliclona collected off Manzamo, Okinawa, Japan as described [8]. The reversible dihydropteridinone BI-D1870, an NTKD-interacting ATP-binding site specific RSK inhibitor was provided by P. Cohen, University of Dundee, Scotland, U.K. [22]. A 10 mM stock of BI-D1870 and MZA were prepared in DMSO and stored at $-80{ }^{\circ} \mathrm{C}$ prior to use in the experiments.

\subsection{Protein kinase activity assays}

Thirty protein kinase activity assays were performed by the Kinase Profiling Screening Service, University of Dundee, as described elsewhere [25,26]. MZA $(1 \mu \mathrm{M})$ was used in all protein kinase assays. Results are presented as a percentage of kinase activity of control incubations (average of duplicate determinations) and are tabulated in Table 1.

\subsection{RSK1 and RSK2 protein kinase assays}

Rat RSK1 (Genbank M99169) and human RSK2 (Genbank NM_004586) protein kinase assays were performed as described in [22] with the following modifications: RSK1 and RSK2 (10 mU) were diluted (20 mM MOPS pH 7.5, $1 \mathrm{mM}$ EDTA, 0.01\% Brij35, 5\% glycerol, 0.1\% $\beta$ mercaptoethanol, $1 \mathrm{mg} / \mathrm{ml}$ BSA) and assayed against KKLNRTLSVA in a final volume of $25 \mu 1$ 
containing $50 \mathrm{mM}$ Na- $\beta$-glycerophosphate $\mathrm{pH}$ 7.5, $0.5 \mathrm{mM}$ EDTA, $30 \mu \mathrm{M}$ substrate peptide, 10 33

$\mathrm{mM}$ magnesium acetate, $0.05 \mathrm{mM}$ [ P-g-ATP] (50-1000 cpm/pmole) and $5 \mu 1 \mathrm{MZA}, \mathrm{BI}-\mathrm{D} 1870$ or vehicle, and incubated for $40 \mathrm{~min}$ at room temperature. The kinase assays were stopped by addition of $5 \mu \mathrm{l}$ of $0.5 \mathrm{M}(3 \%)$ orthophosphoric acid, and $10 \mu \mathrm{l}$ of sample was transferred to labeled P81 filter paper circles and allowed to bind for 30 seconds. Circles were then washed for five minutes with a wash buffer (50 $\mathrm{mM}$ orthophosphoric acid), transferred to scintillation vials containing $5 \mathrm{ml}$ of scintillation cocktail, and read in a scintillation counter.

\subsection{Computational Methods}

\subsubsection{Selection of targets and identification of critical amino acids}

All computations were carried out on quad core Intel 3.0 GHz Xeon processors X5472. The atomic coordinates of Ribosomal S6 Kinase 1 (RSK1) and Ribosomal S6 Kinase 2 (RSK2) were obtained from the Protein Data Bank (PDB). Specifically, complexes with staurosporine (PDB entry 2Z7R) [29], purvalnol A (PDB entry 2Z7S) [29], and AMP-PCP (PDB 2Z7Q) [29] were considered for the N-terminus of RSK1. Similarly, for the N-terminus of RSK2, complexes with afzelin (PDB entry 4EL9) [31], the flavonoid glycoside quercitrin (PDB entry 4GUE) [32], flavonoid glycoside SL0101 (PDB entry 3UBD) [31], and an active conformation of RSK2 (PDB 3G51) [27]. In regards to the C-terminus of RSK1 the apo structure (PDB entry 3RNY) [28] was employed. Finally, representatives of the C-terminal RSK2 were an apo structure (PDB 2QR8) [33], and complexes with (E)-methyl 3-(4-amino-7-(3-hydroxypropyl)-5-p-tolyl-7H-pyrrolo[2,3d]pyrimidin-6-yl)-2-cyanoacrylate (PDB 4D9T) [34] and (E)-tert-butyl-3-(4-amino-7-(3hydroxypropyl)-5-p-tolyl-7H-pyrrolo[2,3-d]pyrimidin-6-yl)-2-cyano- acrylate (PDB 4D9U)[34].

Structural comparisons were carried out within the Maestro interface (Schrodinger, LLC, New York, NY) in order to 1) select the structure that would be similar to the majority of representatives 
of each terminus (Table 2), and 2) identify critical residues that would later serve to define respective binding pockets. Amino acids lining the binding pockets of the crystal RSK1 and RSK2 structures reveal the high degree of similarity (Tables 3a and 3b).

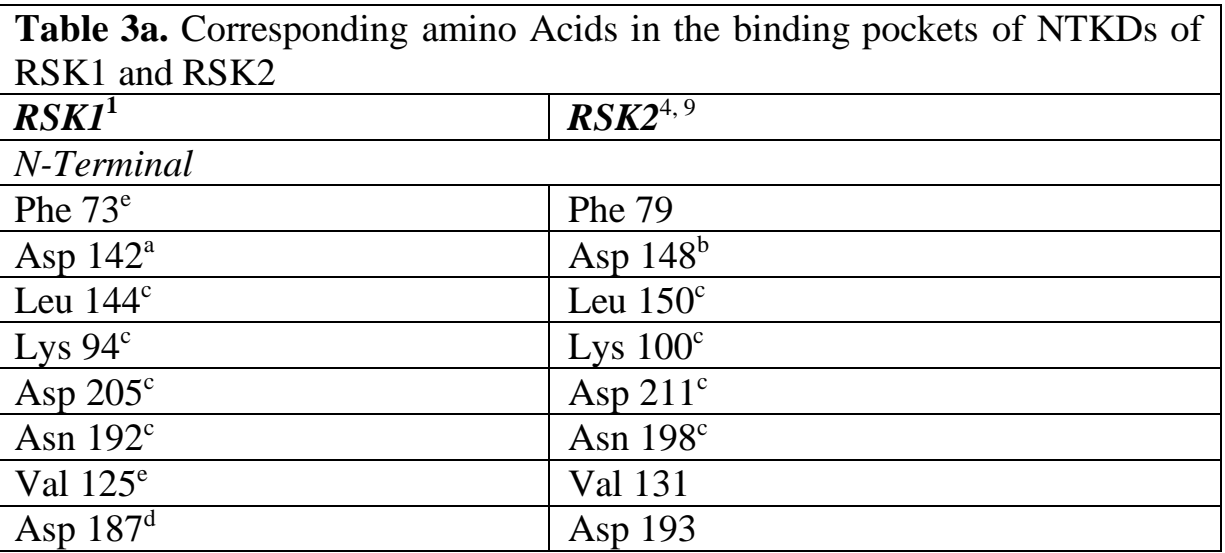

${ }^{\mathrm{a}}$ From [31]. ${ }^{\mathrm{b}}$ Residues in RSK2 that are equivalent to residues reported in reference [31] for RSK1. ${ }^{c}$ Equivalent residues reported in [31,32]. ${ }^{\mathrm{d}}$ Residue in RSK1 corresponding to the one reported for RSK2 [32].

${ }^{\text {e}}$ Reference in RSK1 that is equivalent to the one reported for RSK2 [27].

\begin{tabular}{|c|c|}
\hline RSK1 $^{5}$ & $\mathrm{RSK2}^{7}$ \\
\hline \multicolumn{2}{|l|}{ C-Terminal } \\
\hline Residues around Glu496 & Equivalent residues \\
\hline Leu 691 & Pro 696 \\
\hline Glu 496 & Glu 500 \\
\hline Arg 503 & Arg 507 \\
\hline Asp 499 & Asp 503 \\
\hline Cys 432 & Cys 436 \\
\hline Cys 556 & Cys 560 \\
\hline Thr 489 & Thr 493 \\
\hline ATP-binding cleft & Equivalent residues \\
\hline Lys 447 & Lys 451 \\
\hline Glu 459 & Glu 463 \\
\hline Asp 557 & Asp 561 \\
\hline Asp 535 & Asp 539 \\
\hline Asn 540 & Asn 544 \\
\hline
\end{tabular}




\subsubsection{Protein preparation}

The receptors were prepared by assigning bond orders, adding hydrogens and finding overlaps, followed by hydrogen bond optimization and minimization using the Protein Preparation Wizard. Minimization employed the 'Impref' utility, which runs a series of constrained impact minimizations with gradually decreasing strength of the heavy-atom restraining potential. Two minimizations were initially performed with the heavy-atom restraint potential force constant at 10. In the first minimization, the torsional potential was turned off to improve hydrogen optimization, whereas the second minimization restored the torsional potential. The restraining potential force constant was subsequently reduced to $3,1,0.3$ and 0.1 . If the output structure from a minimization exceeds the specified RMSD threshold, relative to the starting structure, the program stops and returns the structure from the previous minimization. Thus, the RMSD is checked at the end of each round of minimization. Receptor grid generation was subsequently employed with the van der Waals radius scaling factor set to 1.0, and partial charge cut-off at 0.25.

\subsubsection{Docking Glide 5.7}

Glide version $5.7[30,35]$ was used in this study. Glide grids were generated with ligand scaling of 0.8 for the van der Waals radii. Ligands were docked and ranked using standard precision, followed by post-docking minimization. All docking experiments were performed with default settings and 30 poses per ligand were saved for further consideration. Poses were visually inspected and analyzed based on scoring and binding interactions.

\section{Acknowledgements}

This research was completed with institutional support provided by the Office of Research and Sponsored Programs at Midwestern University. Valuable assistance by Midwestern University's 
animal facility and library staff as well as the excellent secretarial assistance of Mrs. Victoria Sears is gratefully acknowledged.

\section{Author Contributions}

A.M.S.M conceived and planned the study and wrote part of the manuscript. M.L.H. supervised the RSK1 and RSK2 protein kinase assays, performed both data and statistical analysis shown in Fig. 2. J.L., J.C., K.C., and C.C. were students in A.M.S.M.'s laboratory, and performed experiments with MZA and rat brain microglia RSK1 and RSK2 expression, and reviewed the manuscript. M.K. completed the computational docking studies depicted in Figures 3-10 and wrote part of the manuscript.

\section{List of abbreviations}

CTKD, C-terminal kinase domain; GSK3, glycogen synthase kinase-3; MZA, Manzamine A; ras-ERK1/2 (extracellular-signal-regulated kinase 1/2) pathway; RSK1, 90kDa ribosomal protein S6 kinase 1; RSK2, 90kDa ribosomal protein S6 kinase 2; NTKD, N-terminal kinase domain; PDB, protein data bank;

\section{Conflicts of Interest}

The authors declare no conflict of interest.

\section{References}

1. Mayer, A.M.S.; Guerrero, J.; Rodríguez, A.D.; Taglialatela-Scafati, O.; Nakamura, F.; Fusetani, N. Marine Pharmacology in 2014-2015: Marine Compounds with Antibacterial, Antidiabetic, Antifungal, Anti-Inflammatory, Antiprotozoal, Antituberculosis, and Antiviral Activities; Affecting the Immune and Nervous Systems, and other Miscellaneous Mechanisms of Action. Marine Drugs 2019, 18(1): 5. 
2. Mayer, A. M. S.; Glaser, K. B.; Cuevas, C.; Jacobs, R. S.; Kem, W.; Little, R. D.; McIntosh, J. M.; Newman, D. J.; Potts, B. C.; Shuster, D. E. The odyssey of marine pharmaceuticals: a current pipeline perspective. Trends Pharmacol. Sci. 2010, 31 (6), 255-265.

3. Skropeta, D.; Pastro, N.; Zivanovic, A. Kinase inhibitors from marine sponges. Mar. Drugs 2011, 9 (10), 2131-2154.

4. Sakai, R.; Higa, T. Manzamine A, a novel antitumor alkaloid from a sponge. J Am. Chem Soc. 1986, 108, 6404-6405.

5. Wahba, A. E.; Fromentin, Y.; Zou, Y.; Hamann, M. T. Acantholactone, a new manzamine related alkaloid with an unprecedented delta-lactone and epsilon-lactam ring system. Tetrahedron Lett. 2012, 53 (47), 6329-6331.

6. Guzman, E. A.; Johnson, J. D.; Linley, P. A.; Gunasekera, S. E.; Wright, A. E. A novel activity from an old compound: Manzamine A reduces the metastatic potential of AsPC-1 pancreatic cancer cells and sensitizes them to TRAIL-induced apoptosis. Invest New Drugs 2011, 29 (5), 777-785.

7. Ang, K. K.; Holmes, M. J.; Kara, U. A. Immune-mediated parasite clearance in mice infected with Plasmodium berghei following treatment with manzamine A. Parasitol. Res. 2001, 87 (9), 715-721.

8. Mayer, A. M.; Hall, M. L.; Lynch, S. M.; Gunasekera, S. P.; Sennett, S. H.; Pomponi, S. A. Differential modulation of microglia superoxide anion and thromboxane B2 generation by the marine manzamines. BMC. Pharmacol. 2005, 5 (1), 6.

9. Yousaf, M.; Hammond, N. L.; Peng, J.; Wahyuono, S.; McIntosh, K. A.; Charman, W. N.; Mayer, A. M.; Hamann, M. T. New manzamine alkaloids from an Indo-Pacific sponge. Pharmacokinetics, oral availability, and the significant activity of several manzamines against HIV-I, AIDS opportunistic infections, and inflammatory diseases. J. Med. Chem. 2004, 47 (14), 3512-3517.

10. Palem, J. R.; Bedadala, G. R.; El Sayed, K. A.; Hsia, S. C. Manzamine A as a novel inhibitor of herpes simplex virus type-1 replication in cultured corneal cells. Planta Med 2011, 77 (1), 46-51.

11. Peng, J.; Kudrimoti, S.; Prasanna, S.; Odde, S.; Doerksen, R. J.; Pennaka, H. K.; Choo, Y. M.; Rao, K. V.; Tekwani, B. L.; Madgula, V.; Khan, S. I.; Wang, B.; Mayer, A. M.; Jacob, M. R.; Tu, L. C.; Gertsch, J.; Hamann, M. T. Structure-activity relationship and mechanism of action studies of manzamine analogues for the control of neuroinflammation and cerebral infections. J. Med. Chem. 2010, 53 (1), 61-76.

12. Zhang, B.; Higuchi, R.; Miyamoto, T.; Van, S. R. Neuritogenic activity-guided isolation of a free base form manzamine A from a marine sponge, Acanthostrongylophora aff. ingens (Thiele, 1899). Chem Pharm. Bull. (Tokyo) 2008, 56 (6), 866-869. 
13. Eguchi, K.; Fujiwara, Y.; Hayashida, A.; Horlad, H.; Kato, H.; Rotinsulu, H.; Losung, F.; Mangindaan, R. E.; de Voogd, N. J.; Takeya, M.; Tsukamoto, S. Manzamine A, a marinederived alkaloid, inhibits accumulation of cholesterol ester in macrophages and suppresses hyperlipidemia and atherosclerosis in vivo. Bioorg. Med. Chem. 2013, 21 (13), 3831-3838.

14. Hamann, M.; Alonso, D.; Martin-Aparicio, E.; Fuertes, A.; Perez-Puerto, M. J.; Castro, A.; Morales, S.; Navarro, M. L.; Del Monte-Millan, M.; Medina, M.; Pennaka, H.; Balaiah, A.; Peng, J.; Cook, J.; Wahyuono, S.; Martinez, A. Glycogen synthase kinase-3 (GSK-3) inhibitory activity and structure-activity relationship (SAR) studies of the manzamine alkaloids. Potential for Alzheimer's disease. J Nat Prod. 2007, 70 (9), 1397-1405.

15. Kallifatidis, G.; Hoepfner, D.; Jaeg, T.; Guzman, E. A.; Wright, A. E. The marine natural product manzamine A targets vacuolar ATPases and inhibits autophagy in pancreatic cancer cells. Mar. Drugs 2013, 11 (9), 3500-3516.

16. Romeo, Y.; Zhang, X.; Roux, P. P. Regulation and function of the RSK family of protein kinases. Biochem. J 2012, 441 (2), 553-569.

17. Heffron, D.; Mandell, J. W. Differential localization of MAPK-activated protein kinases RSK1 and MSK1 in mouse brain. Brain Res. Mol. Brain Res. 2005, 136 (1-2), 134-141.

18. Chandrasena, K.; Hall, M. L.; Mayer, A. M. S. The mechanism of the marine $\beta$-carboline thromboxane $\mathrm{B}_{2}$ inhibitor Manzamine A: possible involvement of rat brain microglia p90 ribosomal S6 kinase 1 (RSK1). FASEB J. 2013, 27, 888.1.

19. Pereira, P. M.; Schneider, A.; Pannetier, S.; Heron, D.; Hanauer, A. Coffin-Lowry syndrome. Eur. J. Hum. Genet. 2010, 18 (6), 627-633.

20. Cho, Y. Y.; Yao, K.; Kim, H. G.; Kang, B. S.; Zheng, D.; Bode, A. M.; Dong, Z. Ribosomal S6 kinase 2 is a key regulator in tumor promoter induced cell transformation. Cancer Res. 2007, 67 (17), 8104-8112.

21. Redman, E. K.; Brookes, P. S.; Karcz, M. K. Role of p90RSK in regulating the Crabtree effect: implications for cancer. Biochem. Soc. Trans. 2013, 41 (1), 124-126.

22. Sapkota, G. P.; Cummings, L.; Newell, F. S.; Armstrong, C.; Bain, J.; Frodin, M.; Grauert, M.; Hoffmann, M.; Schnapp, G.; Steegmaier, M.; Cohen, P.; Alessi, D. R. BI-D1870 is a specific inhibitor of the p90 RSK (ribosomal S6 kinase) isoforms in vitro and in vivo. Biochem. J. 2007, 401 (1), 29-38.

23. Smith, J. A.; Poteet-Smith, C. E.; Xu, Y.; Errington, T. M.; Hecht, S. M.; Lannigan, D. A. Identification of the first specific inhibitor of p90 ribosomal S6 kinase (RSK) reveals an unexpected role for RSK in cancer cell proliferation. Cancer Res. 2005, 65 (3), 1027-1034.

24. Cohen, M. S.; Zhang, C.; Shokat, K. M.; Taunton, J. Structural bioinformatics-based design of selective, irreversible kinase inhibitors. Science 2005, 308 (5726), 1318-1321. 
25. Davies, S. P.; Reddy, H.; Caivano, M.; Cohen, P. Specificity and mechanism of action of some commonly used protein kinase inhibitors. Biochem. J. 2000, 351 (Pt 1), 95-105.

26. Bain, J.; McLauchlan, H.; Elliott, M.; Cohen, P. The specificities of protein kinase inhibitors: an update. Biochem. J. 2003, 371 (Pt 1), 199-204.

27. Malakhova, M.; Kurinov, I.; Liu, K.; Zheng, D.; D'Angelo, I.; Shim, J. H.; Steinman, V.; Bode, A. M.; Dong, Z. Structural diversity of the active N-terminal kinase domain of p90 ribosomal S6 kinase 2. PLoS. One. 2009, 4 (11), e8044.

28. Li, D.; Fu, T. M.; Nan, J.; Liu, C.; Li, L. F.; Su, X. D. Structural basis for the autoinhibition of the C-terminal kinase domain of human RSK1. Acta Crystallogr. D. Biol. Crystallogr. 2012, 68 (Pt 6), 680-685.

29. Ikuta, M.; Kornienko, M.; Byrne, N.; Reid, J. C.; Mizuarai, S.; Kotani, H.; Munshi, S. K. Crystal structures of the N-terminal kinase domain of human RSK1 bound to three different ligands: Implications for the design of RSK1 specific inhibitors. Protein Sci. 2007, 16 (12), 2626-2635.

30. Fisher, T. L.; Blenis, J. Evidence for two catalytically active kinase domains in pp90rsk. Mol. Cell Biol. 1996, 16 (3), 1212-1219.

31. Utepbergenov, D.; Derewenda, U.; Olekhnovich, N.; Szukalska, G.; Banerjee, B.; Hilinski, M. K.; Lannigan, D. A.; Stukenberg, P. T.; Derewenda, Z. S. Insights into the inhibition of the p90 ribosomal S6 kinase (RSK) by the flavonol glycoside SL0101 from the 1.5 A crystal structure of the N-terminal domain of RSK2 with bound inhibitor. Biochemistry 2012, 51 (33), 6499-6510.

32. Derewenda, U.; Artamonov, M.; Szukalska, G.; Utepbergenov, D.; Olekhnovich, N.; Parikh, H. I.; Kellogg, G. E.; Somlyo, A. V.; Derewenda, Z. S. Identification of quercitrin as an inhibitor of the p90 S6 ribosomal kinase (RSK): structure of its complex with the N-terminal domain of RSK2 at 1.8 A resolution. Acta Crystallogr. D. Biol. Crystallogr. 2013, 69 (Pt 2), 266-275.

33. Malakhova, M.; Tereshko, V.; Lee, S. Y.; Yao, K.; Cho, Y. Y.; Bode, A.; Dong, Z. Structural basis for activation of the autoinhibitory C-terminal kinase domain of p90 RSK2. Nat. Struct. Mol. Biol. 2008, 15 (1), 112-113.

34. Serafimova, I. M.; Pufall, M. A.; Krishnan, S.; Duda, K.; Cohen, M. S.; Maglathlin, R. L.; McFarland, J. M.; Miller, R. M.; Frodin, M.; Taunton, J. Reversible targeting of noncatalytic cysteines with chemically tuned electrophiles. Nat. Chem. Biol. 2012, 8 (5), 471-476.

35. Utepbergenov, D.; Derewenda, Z. S. The unusual mechanism of inhibition of the p90 ribosomal S6 kinase (RSK) by flavonol rhamnosides. Biochim. Biophys. Acta 2013, 1834 (7), 1285-1291. 